\title{
EXPOSURE TO NATURAL ENVIRONMENT, SOCIAL ISOLATION AND LONELINESS
}

\author{
Ileana-Loredana Vitalia ${ }^{1 *}$ \\ ${ }^{1}$ University of Pitești, Department of Psychology, Communication Sciences, and Social Assistance, \\ Târgu din Vale, No.1, Pitești, Argeș, Romania
}

\begin{abstract}
Evidence suggests that social isolation and loneliness have became major problems in many countries around the world. Given the numerous physical, mental health problems and economic negative consequences associated with social isolation and loneliness, there is a greater interest for the development of more complex and holistic health strategies to help prevent or mitigate social isolation and loneliness. Natural environment is increasingly recognized as an important component of these strategies. Outdoor natural space (in the form of greenspace, such of parks and street trees, and bluespace, such as rivers and oceans) can reduce the effects of stressors while facilitating social connections, especially emotionally close ties or the sense of community belonging. The present article will consider the results of previous academic studies to discuss the concepts of social isolation and loneliness, the connections between them, and their effects on physical and mental health. This theoretical framework will be used to support the effectiveness of nature-based health interventions for the prevention or the treatment of poor mental health associated with social isolation and loneliness.
\end{abstract}

Keywords: connection with others, mental health, nature

\section{SOCIAL ISOLATION AND LONELINESS}

The complexity of the health and well-being problems associated with social isolation and loneliness (e.g., mortality, worse self-rated physical health, depressive symptoms, psychological distress, greater cognitive decline and impairment, poorer immune system functioning, impaired functional status, vision deficits, and a perceived negative change in the quality of one's life, suicide risk, incident dementia) (Brown and Munson, 2020; Hwang et al., 2020; Nguyen et al., 2020; Taylor, 2019) is the major reason for many organizations from different countries to consider social isolation as a major public health crisis and to develop efficient strategies for preventing and mitigating its negative effects. Their reports document that social isolation is a problem for people from all ages, and underscore the importance of family and friend support networks and ties for understanding isolation.

The present article considers the results of previous academic studies to discuss the concepts of social isolation and loneliness, both as distinct and related aspects with multiple effects on physical and mental health.

The term social isolation is often used to reflect objective structural factors, the level and frequency of one's social interactions (e.g., living alone), whereas the term loneliness is used to reflect 
subjective perceptions (Hwang et al., 2020; Thompson et al., 2020). Current studies typically examine overall social isolation (social isolation from family members, social isolation from friends, living alone, and having limited social participation) (Taylor, 2020).

Loneliness is theorized as a subjective experience, the result of a lack of positive and fulfilling social relationships, as a sense of dissatisfaction an individual has with members of the social network, or a discrepancy between the relationship an individual currently has versus the relationship the individual would like to have (Neves et al., 2019; Taylor, 2020). Loneliness can be experienced as a consequence of being alone and in many cases it is described as a feeling that can be present even when in the company of others. An unfulfilled need for social relationships is considered to be a central factor contributing to loneliness and social isolation (Barbosa Neves et al, 2019).

Research in the field of social isolation (Nguyen et al., 2020) differentiates between objective isolation and subjective isolation. Objective isolation refers to the tangible and quantifiable lack of connections with other people and it is measured by the lack of contact/interaction with social network members. Subjective isolation refers to the perceived lack of closeness between an individual and members of their social network ant it is measured by the lack of emotional closeness to network members. The personal experience of social isolation is very different and it depends on the perceptions of emotional distance and affective features of isolation. A person could describe this experience as being involved in a vast social network but not feeling emotionally close to the persons from this network. This experience is even more dramatic if it involves the family members, because of the protective role of these relations. Family support protects against depressive symptoms, while negative interactions with family members, in the form of arguments and criticisms, are positively associated with mental health problems, suicidal behaviors and psychiatric disorders. In this context, the effects of subjective isolation are mare detrimental to mental health than that of objective isolation.

A large study using a sample of 3570 African Americans (Nguyen et al., 2020) indicates the importance of subjective isolation for psychiatric disorders, and demonstrate that feelings of closeness with family and friends are more significant for mental health than is social contact.

The effects of social isolation on mental health are also described form a culturally based perspective. Studies investigating social isolation among African Americans, Caribbean Black and non-Hispanic White adults evidenced the need to develop a culturally competent care/practice for addressing racial disparities in professional mental health service use and quality of mental healthcare. As described by Lum (Nguyen et al., 2020), this culturally competent practice refers to three distinct domains - cultural awareness, knowledge acquisition, and skill development. From this perspective, the clinical practice becomes more client-centered, and gives the clinician the possibility to have access to the unique experiences and needs of socially marginalized individuals or groups.

Other studies consider the connections between social isolation and loneliness from a developmental and social justice framework across the developmental life span within many different populations (Brown and Munson, 2020). This approach demonstrates the subjective manifestations of social isolation and loneliness across the developmental spectrum, and indicates the importance of clinical and social interventions to combat social isolation (the use of technology, peer support and the facilitation of peer helping relationships, family based interventions).

Taylor (2020) sustains the interaction between social isolation (different types of social isolation) and loneliness (perceptions of loneliness), with the possibility to reinforce and perpetuate each other 
over time. This study measured seven individual indicators that form the social network index of old people: socially isolated from adult children (less than once per month contact with adult children by face to face, telephone, or written/email), socially isolated from other family members (less than once per month contact with other family members by face to face, telephone, or written/email), socially isolated from friends (less than once per month contact with friends by face to face, telephone, or written/ email), no social participation in clubs groups or activities. The results indicates that most types of social isolation are individually and independently associated with greater loneliness in older adults.

\section{THE NEED FOR COMPLEX AND HOLISTIC HEALTH STRATEGIES}

The results of these studies indicate a greater interest for the development of more complex and holistic health strategies to help prevent or mitigate social isolation and loneliness. Some of these strategies are nature-based health interventions. For example, the results form a recents study (Herbolsheimer et al., 2018) the complexity of the reciprocal association between social isolation and depressive symptoms, and reveled the importance of the social network and outdoor environment, since meeting with friends and neighbors and engaging in social activities often takes place outside the house and provide the context for physical activities. Isolation from the family was not related to out-of-home physical activity, while social isolation from friends and neighbors was associated with a decrease in out-of-home physical activity. Exposure to outdoor environments might alter mood, mental well-being, and depressive symptoms, and provide long-term health benefits for older adults. Out-of-home physical activity might be especially beneficial for preventing depression or lowering depressive symptoms. One of the conclusions of this study suggests some efficient interventions addressing social relationships such as: changing natural networks, bringing persons together (other than family members), or opening up facilities for social or recreational group activities. Combining interventions on social relationships with physical activity sought to be a viable option for reducing depressive symptoms.

A large Canadian research (Rugel et al., 2019) highlights the role of natural urban space in facilitating the social connections. The exposure to natural environment increases the sense of community belonging, the development of positive social ties, and provides indirect mental health. Other studies (Araujo et al., 2019) highlights the benefits of performing physical activities in natural environment (green exercise), and advances the idea of a synergistic effect associated with general health. More time spent in natural outdoor activities is associated with more physical activity, higher frequencies of social contacts with neighbors, and higher scores of mental well-being (Kruize et al., 2019).

These studies show that social ties (especially emotionally close ties) have a critically important role in human well-being, particularly among individuals under stress, because they offer expressive (e.g., sense of purpose), informational, and instrumental forms of support that can help to reduce anxiety, increase self-esteem, and provide positive influences regarding health-promoting behaviors, which may be more difficult to maintain during periods of psychological distress (Rugel et al., 2019).

These conclusions became very important in the context of the Covid pandemic. The physical distancing policies introduced to control the disease had dramatic mental health impacts, bringing about increased anxiety and greater social isolation and loneliness (Smith and Lim, 2020). Results from recent studies (Peçanh et al., 2020) indicate a substantial decrease in global physical activity levels during the period of social isolation, with detrimental effects in cardiovascular function. 
There is an urgent need to develop and implement more appropriate programms and interventions for prevention or treatment of the negative physical and psychological effects of the pandemic. Family-level interventions may prove efective for improving health and wellbeing for multiple members of a household (Thompson et al., 2020). In clinical, counselling and psychotherapeutical practice, relaxation, breathing and creative meditation centered on nature helps in activating coping mechanisms (Raban-Motounu, 2019; Vitalia, 2019).

In the same time, population-level interventions and policies that work to strengthen social connections, particularly among groups with increased vulnerability to isolation and illness may be crucial for general health and well being (Barbosa Neves et al., 2019).

\section{REFERENCES}

Araújo, D., Brymer, E., Brito, H., Withagen, R., Davids, K. (2019). The empowering variability of affordances of nature: Why do exercisers feel better after performing the same exercise in natural environments than in indoor environments?, Psychology of Sport \& Exercise 42, 138-145 Retrived from https://doi.org/10.1016/j.psychsport.2018.12.020.

Barbosa Neves, B., Sanders, A., Kokanović, R. (2019). "It's the worst bloody feeling in the world": Experiences of loneliness and social isolation among older people living in care homes. Journal of Aging Studies 49, 74-84. Retrieved from https://doi.org/10.1016/j.jaging.2019.100785

Brown, S., Munson, M.R. (2020). Introduction to the Special Issue on Social Isolation Across the Lifespan. Clinical Social Work Journal, 48, 1-5. Retrieved from https://doi.org/10.1007/s10615-020-00750-3

Herbolsheimer, F., Ungar, N., Peter, R. (2018). Why Is Social Isolation Among Older Adults Associated with Depressive Symptoms? The Mediating Role of Out-of-Home Physical Activity. International Journal of Behavioral Medicine, 25, 649-657. Retrieved from https://doi.org/10.1007/s12529-018-9752-x

Hwang, Tzung-Jeng, Rabheru,K., Peisah, C., Reichman, W., Ikeda, M. (2020). Loneliness and social isolation during the COVID-19 pandemic. Int Psychogeriatr, 1-4. doi: 10.1017/S1041610220000988.

Kruiz, H., van Kamp, I., van den Berg, M., van Kempen, E., Wendel-Vos, W., Ruijsbroek, A., Swart, W., Maas, J., Gidlow, K., Smith, G., Ellis, N., Hurst, G., Masterson, D., Triguero-Mas, M., Cirach, M., Gražulevičienè, R., van den Hazel, P., Nieuwenhuijsen, M. (2020). Exploring mechanisms underlying the relationship between the natural outdoor environment and health and well-being - Results from the PHENOTYPE project. Environment International 134, 105173. Retrieved from https://doi.org/10.1016/j.envint.2019.105173

Nguyen, A.N., Taylor, R.J., Taylor, H.O., Chatters, L.M. (2029). Objective and Subjective Social Isolation and Psychiatric Disorders Among African Americans. Clinical Social Work Journal, 48, 87-98. Retrieved from https://doi.org/10.1007/s10615-019-00725-z

Peçanh, T., Hamilton Roschel, K.F., Gualano, B. (2020). Social isolation during the COVID-19 pandemic can increase physical inactivity and the global burden of cardiovascular disease. American Journal of Physiology Heart and Circulatory Physiology, 318(6), H1441-H1446. doi: 10.1152/ajpheart.00268.2020

Raban-Motounu, N. (2019). Relaxation and creative meditation centered on nature helps in activating coping mechanisms. Current Trends in Natural Sciences, 8(16), 190-195. Retrieved from https://natsci.upit.ro/issues/2019/volume-8-issue-16/

Rugel, E. J., Carpiano, R.M. , Henderson, S.B., Brauer, M. (2019). Exposure to natural space, sense of community belonging, and adverse mental health outcomes across an urban region. Environmental Research 171, 365-377, Retrived from https://doi.org/10.1016/j.envres.2019.01.034.

Smith BJ, Lim MH (2020). How the COVID-19 pandemic is focusing attention on loneliness and social isolation. Public Health Res Pract., 30(2):e3022008. Retrieved from https://doi.org/10.17061/phrp3022008

Taylor, H.O. (2020). Social Isolation's Infuence on Loneliness Among Older Adults. Clinical Social Work Journal, 48, 140-151. Retrieved from https://doi.org/10.1007/s10615-019-00737-9

Thompson, T., Rodebaugh, T.L., Bessaha, M.J., Sabbath, E.L. (2020). The Association Between Social Isolation and Health: An Analysis of Parent-Adolescent Dyads from the Family Life, Activity, Sun, Health, and Eating Study. Clinical Social Work Journal. 48, 18-24. Retrieved from https://doi.org/10.1007/s10615-019-00730-2

Vitalia, I.L. (2019). The breath - the experience of being present. Current Trends in Natural Sciences, 8(16), 196-199. Retrieved from https://natsci.upit.ro/issues/2019/volume-8-issue-16/ 